\title{
EFFECT OF USING CUSTOM-MADE TOOTHBRUSH ON THE GINGIVAL HEALTH OF REMOVABLE PARTIAL DENTURE ABUTMENTS AND DENTURE HYGIENE IN PATIENTS WITH RHEUMATOID ARTHRITIS
}

\author{
Enas E. Mesallum*
}

\begin{abstract}
Purpose: The aim of this study was to investigate the effect of using custom-made toothbrushes in the gingival health of removable partial denture abutments and denture hygiene in Rheumatoid Arthritis patients.

Materials and Methods: Twenty Arthritis female participants with mild to moderate manual dexterity were recruited for this crossover study. All patients received mandibular unmodified Kennedy class II removable partial dentures of the same design. Custom-made brushes were made by adapting silicone putty to the brush handle to conform to the patient's dexterity. The participants were divided randomly into two groups: Group I: started with conventional toothbrush/followed by custom-made brush, group II: started with custom-made brush/ followed by conventional toothbrush. Plaque index PI and gingival index GIwere recorded for the main abutments as a base line, then after 2 and 4 weeks. Denture plaque index DPI was evaluated 2 weeks after denture delivery, and at the end of the 4-week period for each brush group.
\end{abstract}

Results: The conventional toothbrush group showed statistically significant higher values of the mean PI, and the mean GI after 2 as well as 4 weeks. However, both groups show significant reduction in the GI after the 4 weeks period. Conventional brush group revealed significantly higher mean DPI values than the custom-made brush group after the 4-week period.

Conclusion: Within the limitations of this study, it can be concluded that using custom-made toothbrush for Rheumatoid Arthritis patients caused significant improvement in plaque control, gingival health of partial denture abutments, and denture hygiene.

* Oral and Maxillofacial Prosthodontic Department, Faculty of Dentistry, King Abdulaziz University, Jeddah, Saudi Arabia 


\section{INTRODUCTION}

Rheumatoid arthritis (RA) is a chronic destructive inflammatory autoimmune disease characterized by the accumulation and persistence of an inflammatory infiltrate in the synovial membrane that leads to synovitis and destruction of the joint architecture. $50 \%$ of the risk for development of rheumatoid arthritis is attributable to genetic factors. The disorder is most typical in women and elderly people. $^{(1)}$

RA has many pathologic features similar to that of periodontitis (PD). ${ }^{(2)}$ Cytokines such interleukin$1 b$ (IL-1b), interleukin-6 (IL-6), and tumor necrosis factor-a (TNF-a) play a crucial role in both PD and RA. ${ }^{(2,3)}$ Associations between RA and PD have been detected in different studies. ${ }^{(4,5,6)}$ Moreover, patients with advanced RA are at an increased risk of suffering from more severe periodontal problems compared with patients without RA. ${ }^{(5,7,8)}$

Another important point in respect to arthritis is its effect on the TMJ leading to pain and limited mouth opening. ${ }^{(9)}$ Furthermore, reduction in masticatory performance and the maximum bite force has been observed in these patients. ${ }^{(10)}$ However, a recent study reported that the mastication of elders with RA can be improved by the insertion of wellfitted removable partial dentures RPDs, minimizing the effects of the disease on the stomatognathic system $^{\cdot(11)}$

On the other hand, some studies revealed that RPDs generated more plaque accumulation and gingivitis around direct abutments, in comparison to non-abutments. ${ }^{(12,13)}$ This means that partially edentulous arthritis patients wearing RPDs could have multifactorial risk factors that may cause gingivitis and periodontitis especially around abutment teeth. Accordingly, such patients should keep meticulous oral hygiene. Unfortunately, oral hygiene may be impaired in these patients due to the manual disability as RA commonly affects the proximal interphalangeal and metacarpophalangeal joints which complicate the proper use of the conventional toothbrush. ${ }^{(14)}$

For patients with such reduced manual dexterity improving the grip of the toothbrush by various methods is described in the literature. ${ }^{(14)}$ Several studies considered that using silicone putty impression material molded to the brush handle is the simplest and the most successful technique as it prevents the brush from rotating because of the individual's reduced grip strength. ${ }^{(14,15)}$ But the effect of this brush in improving the oral hygiene of RA patients especially after wearing RPDs still unknown.

To the best of my knowledge, no study has assessed the oral hygiene in partially edentulous RA patients wearing removable prosthesis using a modified toothbrush. Thus, the aim of this study was to investigate the effect of using custommade toothbrushes in the gingival health of RPD abutments and denture hygiene in arthritis patients.

\section{MATERIALS AND METHODS}

\section{Study design and population}

This study was a single-blind, cross over randomized controlled clinical study compared the effect of using custom-made toothbrush and conventional manual toothbrush on the gingival health of RPD abutments and denture hygiene among arthritis participants. Study was conducted between January 2019 and August 2020.

Twenty Arthritis female participants between the ages of 55-72 years were recruited for this studs from King Abdulaziz University, Faculty of Medicine, Rheumatology Clinic. It was necessary that subjects met the following selection criteria: Arthritis participants with mild to moderate manual dexterity (Fig.1), having completely dentate maxillary arches and, mandibular Kennedy class I partially edentulous arches with the first or the second premolar as the last standing abutments, and 


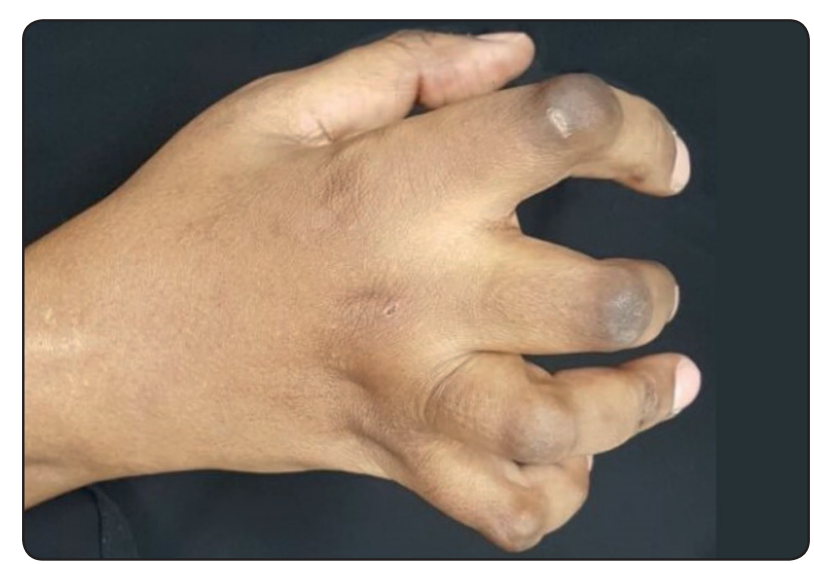

Fig. (1): Arthritis patient`s manual dexterity

more than $8 \mathrm{~mm}$ distance between the free gingival margin and the floor of the mouth. Participants with any systemic diseases other than arthritis such as (diabetes, cardiopathy, hypertension), recent extractions, and periodontal or antibiotic therapy within the previous 3 months were excluded. At the screening visit, subjects were asked to read and sign a written informed consent.

\section{Prosthetic procedures}

For all the included participants RPDs with Cobalt-Chromium frameworks were constructed according to the conventional prosthetic procedures. The RPD design was consisted of reverse Aker clasps as direct retainers on the main abutments. The indirect retainers were cingulum rests on the canines when the last abutments were the first premolars, or distal rest on the first premolar when the last abutments were the second premolars. Lingual bar major connector was used for all the cases. Before prosthetic rehabilitation, all the selected participants received supra and subgingival scaling and root planning, followed by proper oral hygiene instructions.

\section{Clinical measurements}

The day of prosthesis delivery plaque index PI and gingival index GI were recorded for the main abutments as a base line record.
Plaque Index of Silness and Loe were scored as follows: ${ }^{(16)}$

$0=$ No plaque

$1=$ plaque adhering to the free gingival margin and adjacent area of the tooth. The plaque may be seen only after application of disclosing solution or by using the probe on the tooth surface.

$2=$ Moderate accumulation of soft deposits within the gingival pocket, or the tooth and gingival margin which can be seen with the naked eye.

$3=$ Abundance of soft matter within the gingival pocket and/or on the tooth and gingival margin.

Loe \& Silness Gingival Index were scored as follows: ${ }^{(17)}$

Score $0=$ Normal gingiva .

Score 1 = Mild inflammation - slight change in color, slight edema. No bleeding on probing.

Score 2 = Moderate inflammation - redness, edema, glazing. Bleeding on probing.

Score 3 = Severe inflammation - marked redness and edema, ulceration. Tendency toward spontaneous bleeding.

Subjects were randomized to the treatment sequence by using the closed envelope method into two groups:

Group I: start with conventional toothbrush/ followed by custom-made brush

Group II: start with custom-made brush/ followed by conventional toothbrush

All participants were given a new conventional soft-bristle toothbrush of the same brand (Al Felaij Junior, Al Felaij Co. Muscat, Oman); custom-made toothbrushes were fabricated for each patient at the day of the prosthesis delivery. 


\section{Custom-made toothbrush Construction}

Silicone putty impression material (C-Silicone Bonasil Putty, MP Co. Athens, Greece) was molded and adapted to the brush handle then griped by the patient's hand to conform to the degree of the participant's dexterity (Fig. 2).

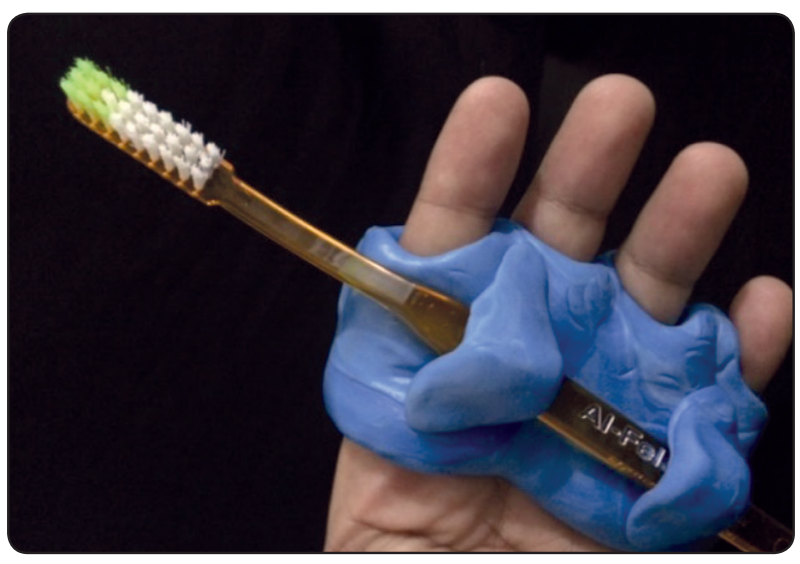

Fig. (2): Silicone putty attached to the toothbrush to conform to the patient's dexterity

Participants were trained for using their respective brushes according to the Bass brushing technique twice daily using the same type of fluoridated toothpaste. They were instructed to brush their dentures after meals using their brushes under tap water, to store the dentures overnight in tap water, and to avoid using other oral hygiene aids, mouth washes, or denture cleansers during the entire study period.

Both groups were instructed to use the first brush for 4 weeks, and then they returned to use their own brushes for 2 weeks washout period, after that they switched to use the other brush according to the assigned treatment sequence for another 4 weeks. The clinical indices of GI and PI were recorded every 2 weeks for each brush group by a calibrated hygienist who was blinded to the intervention. The indices were recorded at the same method as the base line.

\section{Evaluation of denture hygiene}

The state of denture hygiene was evaluated 2 weeks after denture delivery, and at the end of the 4-week period for each brush group. The accumulation of plaque, and calculus of the RPD were measured according to Jeganathan et $\mathrm{al}^{(18)}$ and Ribeiro et al. ${ }^{(19)}$ The prostheses were disclosed with erythrocin solution and the following surfaces were observed: inner surface clasp (Area 1), inner surface major connector (Area 2), artificial acrylic resin teeth (Area 3) and denture base (Area 4). Plaque, and calculus on the surfaces of the dentures were scored as follows: $0=$ no plaque and calculus; $1=$ light plaque and calculus (25\% of the fitting surface covered); 2 = moderate plaque, calculus and stain (26-50\% of the fitting surface covered); $3=$ heavy plaque, calculus and stain (51-100\% of the fitting surface covered).

\section{Statistical Analysis}

All indices were non-parametric data and were presented as median and range values. Since the study is a cross-over design, Wilcoxon signedrank test was used for comparisons between the two groups. Friedman's test was used to study the changes by time within each group. Dunn's test was used for pair-wise comparisons when Friedman's test is significant. The significance level was set at $\mathrm{P} \leq 0.05$. Statistical analysis was performed with IBM SPSS Statistics for Windows, Version 23.0. Armonk, NY: IBM Corp.

\section{RESULTS}

\section{Demographic data}

The present study was conducted on 20 patients; all were females. The mean and standard deviation values for age were $68.5 \pm 8.5$ years old with a minimum of 55 and a maximum of 72 years old.

\section{A) Plaque Index (PI)}

The conventional brush showed a statistically significant higher median PI than the custom-made brush after 2 as well as 4 weeks. ( $P$-value $<0.001$, Effect size $=0.888)$ and $(P$-value $<0.0001$, Effect size $=0.890)$ respectively. 
Concerning the changes by time in conventional brush group; there was no statistically significant change in the median PI by time. While in the custom-made brush group; there was a statistically significant decrease in the median PI after 2 weeks followed by a non-statistically significant change in median PI from 2 to 4 weeks. However, the median PI after 4 weeks revealed a statistically significant lower value compared to base line.

\section{B) Gingival Index (GI)}

Similarly, the conventional brush demonstrated a statistically significant higher median GI than custom-made brush after 2 as well as 4 weeks; $(P$-value $<0.001$, Effect size $=0.880)$ and $(P$-value $<0.0001$,
Effect size $=0.888)$, respectively.

However, both groups presented a statistically significant decrease in the median GI after 2 weeks followed by a non-statistically significant change in median GI from 2 to 4 weeks. The median GI after 4 weeks showed statistically significant lower value compared to base line in both groups.

\section{C) Overall Denture Plaque Index (DPI)}

After 2 as well as 4 weeks; conventional brush showed statistically significant higher median overall Denture Plaque Index than the custom-made brush $(P$-value $<0.001$, Effect size $=0.917)$ and $(P$-value $<0.001$, Effect size $=0.888)$, respectively.

TABLE (1): Descriptive statistics and results of Wilcoxon signed-rank test for comparison between Plaque index (PI) in the two groups and Friedman's test for the changes by time within each group

\begin{tabular}{|c|c|c|c|c|c|c|}
\hline \multirow{2}{*}{ Time } & \multicolumn{2}{|c|}{ Conventional brush } & \multicolumn{2}{|c|}{ Custom-made brush } & \multirow{2}{*}{$P$-value } & \multirow{2}{*}{ Effect size $(r)$} \\
\hline & Median & Range & Median & Range & & \\
\hline Base line & 2.5 & $2.08-2.92$ & $2.63^{\mathrm{A}}$ & $2.26-3$ & 0.186 & 0.296 \\
\hline 2 weeks & 2.56 & $2.06-3.06$ & $2.19^{\mathrm{B}}$ & $1.9-2.48$ & $<0.001 *$ & 0.888 \\
\hline 4 weeks & 2.48 & $2.1-2.86$ & $2.05^{\text {в }}$ & $1.75-2.35$ & $<0.001 *$ & 0.890 \\
\hline$P$-value & \multicolumn{2}{|c|}{0.051} & \multicolumn{2}{|c|}{$<0.001 *$} & & \\
\hline Effect size $(r)$ & \multicolumn{2}{|c|}{0.165} & \multicolumn{2}{|c|}{1.000} & & \\
\hline
\end{tabular}

*: Significant at $P \leq 0.05$, Different superscripts in the same column are significantly different

TABLE (2): Descriptive statistics and results of Wilcoxon test for comparison between Gingival Index (GI) in the two groups and Friedman's test for the changes by time within each group

\begin{tabular}{|c|c|c|c|c|c|c|}
\hline \multirow{2}{*}{ Time } & \multicolumn{2}{|c|}{ Conventional brush } & \multicolumn{2}{c|}{ Custom-made brush } & \multirow{2}{*}{ P-value } & Effect size $(r)$ \\
\cline { 2 - 6 } & Median & Range & Median & Range & \\
\hline Base line & $1.07^{\mathrm{A}}$ & $0.85-1.29$ & $1.08^{\mathrm{A}}$ & $0.95-1.21$ & 0.290 & 0.237 \\
\hline 2 weeks & $0.99^{\mathrm{B}}$ & $0.77-1.21$ & $0.83^{\mathrm{B}}$ & $0.71-0.95$ & $<0.001^{*}$ & 0.880 \\
\hline 4 weeks & $0.94^{\mathrm{B}}$ & $0.74-1.14$ & $0.73^{\mathrm{B}}$ & $0.54-0.92$ & $<0.001^{*}$ & 0.888 \\
\hline$P_{\text {-value }}<0.001^{*}$ & \multicolumn{2}{|c|}{$<0.001^{*}$} & & \\
\hline Effect size $(r)$ & \multicolumn{2}{|c|}{0.999} & \multicolumn{2}{|c|}{0.978} & & \\
\hline
\end{tabular}

*: Significant at $P \leq 0.05$, Different superscripts in the same column are statistically significantly different 
Regarding the changes by time in both groups; there was a statistically significant change in the median overall Denture Plaque Index by time $(P$ value $<0.001$, Effect size $=0.650)$ and $(P$-value $<0.001$, Effect size $=0.330)$, respectively.

Pair-wise comparisons between the time periods in the conventional brush group showed that there was a statistically significant increase in median overall Denture Plaque Index after 2 weeks fol- lowed by a non-statistically significant change in the median DPI from 2 to 4 weeks.

Pair-wise comparisons between the time periods in the custom-made brush group revealed that there was no statistically significant change in the median overall Denture Plaque Index after 2 weeks followed by a statistically significant decrease in the median DPI from 2 to 4 weeks.

TABLE (3): Descriptive statistics and results of Wilcoxon signed-rank test for comparison between Denture Plaque Index in the two groups and Friedman's test for the changes by time within each group.

\begin{tabular}{|c|c|c|c|c|c|c|c|}
\hline \multirow{6}{*}{ Area } & \multirow{2}{*}{ Time } & \multicolumn{2}{|c|}{ Conventional brush } & \multicolumn{2}{|c|}{ Custom-made brush } & \multirow{2}{*}{$P$-value } & \multirow{2}{*}{ Effect size (r) } \\
\hline & & Median & Range & Median & Range & & \\
\hline & 2 weeks & $2^{\mathrm{A}}$ & $1-2$ & $1^{\mathrm{A}}$ & $1-1$ & $<0.001 *$ & 0.837 \\
\hline & 4 weeks & $2^{\mathrm{A}}$ & $1-2$ & $0^{\text {в }}$ & $0-2$ & $0.008^{*}$ & 0.592 \\
\hline & $P$-value & \multicolumn{2}{|c|}{$0.001 *$} & \multicolumn{2}{|c|}{$0.001 *$} & & \\
\hline & Effect size $(r)$ & \multicolumn{2}{|c|}{0.370} & \multicolumn{2}{|c|}{0.350} & & \\
\hline \multirow{4}{*}{$\begin{array}{l}\text { Inner major } \\
\text { connector }\end{array}$} & 2 weeks & $2^{\mathrm{A}}$ & $1-2$ & $1^{\mathrm{A}}$ & $0-1$ & $<0.001^{*}$ & 0.806 \\
\hline & 4 weeks & $2^{\mathrm{A}}$ & $1-2$ & $0^{\text {в }}$ & $0-1$ & $<0.001 *$ & 1.000 \\
\hline & $P$-value & \multicolumn{2}{|c|}{$0.001 *$} & \multicolumn{2}{|c|}{$<0.001 *$} & & \\
\hline & Effect size $(r)$ & \multicolumn{2}{|c|}{0.350} & \multicolumn{2}{|c|}{0.488} & & \\
\hline \multirow{4}{*}{$\begin{array}{l}\text { Artificial denture } \\
\text { teeth }\end{array}$} & 2 weeks & $2^{\mathrm{A}}$ & $1-2$ & $1^{\mathrm{A}}$ & $1-2$ & $0.014^{*}$ & 0.548 \\
\hline & 4 weeks & $2^{\mathrm{A}}$ & $1-2$ & $0^{\text {в }}$ & $0-1$ & $0.001 *$ & 0.732 \\
\hline & $P$-value & \multicolumn{2}{|c|}{$<0.001 *$} & \multicolumn{2}{|c|}{$<0.001 *$} & & \\
\hline & Effect size $(r)$ & \multicolumn{2}{|c|}{0.650} & \multicolumn{2}{|c|}{0.488} & & \\
\hline \multirow{4}{*}{ Fitting surface } & 2 weeks & $2^{\mathrm{A}}$ & $1-2$ & $2^{\mathrm{A}}$ & $1-2$ & 0.782 & 0.062 \\
\hline & 4 weeks & $1^{\text {в }}$ & $1-3$ & $1^{\text {в }}$ & $1-2$ & $0.008 *$ & 0.592 \\
\hline & $P$-value & \multicolumn{2}{|c|}{$0.001 *$} & \multicolumn{2}{|c|}{$0.002 *$} & & \\
\hline & Effect size $(r)$ & \multicolumn{2}{|c|}{0.350} & \multicolumn{2}{|c|}{0.318} & & \\
\hline \multirow{4}{*}{ Overall } & 2 weeks & $1.5^{\mathrm{A}}$ & $1.25-2$ & $1^{\mathrm{A}}$ & $1-1.5$ & $<0.001 *$ & 0.917 \\
\hline & 4 weeks & $1.5^{\mathrm{A}}$ & $1.25-1.5$ & $1^{\mathrm{A}}$ & $0.5-1.25$ & $<0.001 *$ & 0.888 \\
\hline & $P$-value & \multicolumn{2}{|c|}{$<0.001 *$} & \multicolumn{2}{|c|}{$0.001 *$} & & \\
\hline & Effect size $(r)$ & \multicolumn{2}{|c|}{0.650} & \multicolumn{2}{|c|}{0.330} & & \\
\hline
\end{tabular}




\section{DISCUSSION}

This study aimed to compare the effectiveness of conventional and custom-made toothbrushes for the gingival health and denture hygiene of Rheumatoid Arthritis patients. This is because Rheumatoid Arthritis is known to be associated with manual disability that renders keeping efficient oral and denture cleaning is difficult to those patients. Several studies revealed that Arthritis is associated with periodontal disease. ${ }^{(4,5,20)}$

As the performance of the manual toothbrush is greatly determined by the degree of manual dexterity and patient's skills, putty elastomeric material is applied to the handle of the toothbrush to obtain fingerprints conforming to the patient's dexterity to facilitate brushing.

All patients were females to exclude the variability in motor skills between males and females. The selected cases were all with maxillary dentate arches opposing mandibular class I and all received a RPD of the same design to reduce the study variables. Gingivally approaching clasps were not used to avoid any traumatizing effects to the gingival tissues.

According to the literature, the performance of the toothbrushes has been evaluated in the form of recording the PI as dental plaque is the primary etiological agent in dental caries and periodontal diseases. ${ }^{(21)}$ Similarly, gingival index is usually used in the clinical trials to investigate the presence and the severity of plaque induced gingivitis. ${ }^{(22)}$

The reduction in gingival scores in both groups after the 4 weeks period can be attributed to the compliance of patients, and the constant reinforcement of oral hygiene instructions given to them in each visit.

However, the custom-made brush caused more reduction in GI, and PI. As there was a statistically significant reduction of both indices in the custommade brush group after the 4 weeks period as compared to the base line.
This could be explained by the improved gripping strength of patients using the custom brush with the silicone putty conforming to their hands and allow them to perform more efficient cleaning and thereby improve their plaque removal efficiency as compared to the conventional brush.

This is in accordance with studies that compared between electric and manual toothbrush for special need patients, ${ }^{(23)}$ and for neuromuscular disability ${ }^{(24)}$. These studies demonstrated that the ease of using the brush with lesser effort caused a significant improvement in plague and gingival scores as compared to the manual toothbrush.

Previous studies found that wearing RPDs is important to improve mastication and to minimize effects of the RA on the stomatognathic system ${ }^{(11)}$. However, improper denture hygiene can lead to caries and periodontitis. Moreover, some studies correlated between periodontal treatment and RA as periodontal treatment aids in the control of RA related inflammation markers associated with RA. ${ }^{(8,22)}$ which make denture hygiene in those patients more critical.

Denture hygiene was explored using denture plaque index which is a simple procedure that aims to identify the amount of plaque accumulated over different denture surfaces. ${ }^{(16)}$

Although denture cleaning was ascertained to be followed daily by patients in both groups, thereby removing all visible plaque, none of the dentures were free from plaque (score 0). An average denture plaque score of 1.5 , and 1 on a 0 to 4 scale was observed at the end of the test periods for conventional and custom-made brushes respectively. Most of the denture sites were less than $50 \%$ covered with plaque (scores 1 or 2 ).

The present study demonstrated that patients using custom-made brushes showed a significant overall reduction in denture plaque index values from 2 weeks to the 4 week period. On the other 
hand, no significant change has revealed in the conventional brush group in the same time period. This also reflects that the custom-made brush facilitates proper denture cleaning as compared to the conventional brush.

This study has some limitations. The 4-week observation period may have been too short to fully evaluate the impact of custom-made toothbrush on Arthritis patients, and the sample size was small. Longer term studies including more patients are required to evaluate the advantage of the custommade toothbrush in this respect. However, the findings of this study revealed that the use of a custom-made toothbrush with putty handle for patients with RA is a simple procedure that improves the patient's oral hygiene thus stimulates greater independence and give those patients better emotional conditions.

\section{CONCLUSION}

Within the limitations of this study, it can be concluded that using custom-made toothbrush that conforms to the degree of manual dexterity in Rheumatoid arthritis patients caused significant improvement in plaque control, gingival health of partial denture abutments, and denture hygiene.

\section{REFERENCES}

1. David L S, Frederick W, Tom W J. Rheumatoid arthritis Lancet. 2010; (25): 1094-1108.

2. McInnes IB, Schett G. Cytokines in the pathogenesis of rheumatoid arthritis. Nat Rev Immunol 2007; (7):429-442.

3. Liu YC, Lerner UH, Teng YT. Cytokine responses against periodontal infection: protective and destructive roles. Periodontol 2000 2010; (52):163-206.

4. Persson GR. Rheumatoid arthritis and periodontitisinflammatory and infectious connections. Review of the literature. J Oral Microbiol 2012; (4): 1-16

5. Mahajan V, Vaish S, Dodwad V, Nagpal S. InterRelationship between Rheumatoid Arthritis and Periodontal Disease: A Case Control Study Journal of Dental Specialities, 2014; 2,(1):4-14
6. Payne JB, Golub LM, Thiele GM, Mikuls TR. The link between periodontitis and rheumatoid arthritis: a periodontist's perspective. Curr Oral Health Rep 2015; (2):20-29

7. Pablo P, Chapple IL, Buckley CD, Dietrich T. Periodontitis in systemic rheumatic diseases. Nat Rev Rheumatol 2009; (5) :218-224

8. De Smit MJ, Westra J, Brouwer E, Janssen KM, Vissink A, van Winkelhoff AJ. Periodontitis and rheumatoid arthritis: what do we know? J Periodontol 2015; (86):1013-1022.

9. Andrade KM, Alfenas BFM, Campos $\mathrm{CH}$, Rodrigues Garcia RCM. Mandibular movements in older people with rheumatoid arthritis. Oral Surg Oral Med Oral Pathol Oral Radiol. 2017; 123(5): 153-159

10. Bessa-Nogueira RV, Vasconcelos BC, Duarte AP, Góes PS, Bezerra TP. Targeted assessment of the temporomandibular joint in patients with rheumatoid arthritis. J Oral Maxillofac Surg. 2008; (66):1804-1811.

11. Andrade KM , Alfenas BF. M, Rodrigues GarciaR. CM. Influence of removable prostheses on mastication in elderly subjects with rheumatoid arthritis. J Oral Rehabil. 2018; (45):295-300.

12. Preshawab A P., Wallsa W.G., Jakubovicsa N.S, Moynihanac, Jepsona N.J et al. Association of removable partial denture use with oral and systemic health. Journal of Dentistry 2011; (11): 711-719

13. Adriana P.C, Kassia CD, Ana L'1lian CL, Camila MB, Machado R, et al. Periodontal Conditions of Abutments and Non-Abutments in Removable Partial Dentures over 7 Years of Use, Journal of Prosthodontics 2017; (26): 644-649

14. Dougall A. and Fiske J. Access to special care dentistry, part 4. Education. British Dental Journal 2008; (3): 205-216

15. Fiske J, Dickinson C, Boyle C, Rafique S, Burke M. Managing the health of patients with physical disabilities. In Special care dentistry. pp 9-26. London: Quintessence Publishing, 2007

16. Silness, J. and Loe, H. (1964) Periodontal Disease in Pregnancy. II. Correlation between Oral Hygiene and Periodontal Condition. Acta Odontologica Scandinavica, 22, 121-135.

17. Löe H: The Gingival Index, the Plaque Index and the Retention Index Systems. Journal of Periodontology. Volume38 (6), 610-616, 1967 
18. Jeganathan S, Thean HP, Thong KT, Chan YC,Singh M. A clinically viable index for quantifying denture plaque. Quintessence Int 1996; 27(8): 569-573.

19. Ribeiro1 DG, Pavarina1 AC, Giampaolo1 ET, Machado1, AL Jorge $\mathrm{JH}$ et al. Effect of oral hygiene education and motivation on removable partial denture wearers: longitudinal study. Gerodontology 2009; (26):150-156

20. Kaur S, White S, Bartold PM .Periodontal disease and rheumatoid arthritis: a systematic review. J Dent Res 2013; (92):399-408

21. Stenudd C, Nordlund A, Ryberg M, Johansson I, Källestål $\mathrm{C}$, et al. The association of bacterial adhesion with dental caries. J Dent Res. 2001;(80):2005-2010
22. Yang NY, Wang CY, Chyuan IT, Wu KG, Tu UK, et al. Significant association of rheumatoid arthritis-related inflammatory markers with non-surgical periodontal therapy Journal of the Formosan Medical Association 2018; (117):1003-1010

23. Mayuri Vajawat, P. C. Deepika, Vijay Kumar, and P. Rajeshwari_A clinicomicrobiological study to evaluate the efficacy of manual and powered toothbrushes among autistic patients Contemp Clin Dent. 2015; 6(4): 500-504

24. Tokuhei Ikeda, Kunio Y, Kazuya T, Chiho I, Kiyonobu K, Kazuhiko K, Shirou S. Effectiveness of electric toothbrushing in patients with neuromuscular disability: A randomized observer-blind crossover trial. Spec Care Dentist 2016; 36(1): 13-17 